\title{
Numerical study of the isotope effect in underdoped high-temperature superconductors: Calculation of the angle-resolved photoemission spectra.
}

\author{
A.S. Mishchenko ${ }^{1,2}$ and N. Nagaosa ${ }^{1,3}$ \\ ${ }^{1}$ CREST, Japan Science and Technology Agency (JST), AIST, 1-1-1, Higashi, Tsukuba 305-8562, Japan \\ ${ }^{2} R R C$ "Kurchatov Institute", 123182, Moscow, Russia \\ ${ }^{3}$ CREST, Department of Applied Physics, The University of Tokyo, 7-3-1 Hongo, Bunkyo-ku, Tokyo 113, Japan
}

\begin{abstract}
We present a numerical study of the isotope effect on the angle resolved photoemission spectra (ARPES) in the undoped cuprates. By the systematic-error-free Diagrammatic Monte Carlo method, the Lehman spectral function of a single hole in the $t t^{\prime} t^{\prime \prime}-J$ model in the regime of intermediate and strong couplings to optical phonons is calculated for normal and isotope substituted systems. We found that the isotope effect is strongly energy-momentum dependent, and is anomalously enhanced in the intermediate coupling regime while it approaches to that of the localized hole model in the strong coupling regime. We predict the strengths of effect as well as the fine details of the ARPES lineshape change. Implications to the doped case are also discussed.
\end{abstract}

PACS numbers: 71.10.Fd, 71.38.-k, 79.60.-i, 02.70.Ss

It is a subject of considerable debates for many years whether the electron-phonon interaction (EPI) is essential for the physics of undoped and heavily underdoped high $T_{c}$ superconductors $[1,2,3,4,4,5,6]$. Early study of the small isotope effect (IE) on $T_{c}$ at optimal doping together with the no prominent phonon features in the temperature dependence of the resistivity $\rho(T)$ had led to the conclusion that the phonons are a by-player in high $T_{c}$ cuprates. However, the roles of EPI have been recently studied intensively in terms of the neutron scattering, Raman scattering, the IE on $T_{c}$ and the superfluid density $\rho_{s}$, and ARPES [4]. Especially ARPES provides the direct information on the single-particle Green function, which has revealed the "kink" structure of the electron energy dispersion $40-70 \mathrm{meV}$ below the Fermi energy. The origin of this kink is naturally interpreted as the coupling to some bosonic modes. The magnetic resonance mode and the phonon modes are the two major candidates, and the IE on ARPES should be the smokinggun experiment to distinguish between these two. Gweon et al. 7] performed the ARPES experiment on $\mathrm{O}^{18}$ replaced Bi2212 at optimal doping and found an appreciable IE, which however can not be explained within the conventional weak-coupling Migdal-Eliashberg theory. Namely the change of the spectral function due to $\mathrm{O}^{18}$-replacement has been observed at higher energy region beyond the phonon energy $(\sim 60 \mathrm{meV})$. This is in sharp contrast to the weak coupling theory prediction, i.e., the IE should occur only near the phonon energy. Hence the IE in optimal Bi2212 remains still a puzzle. On the other hand, the ARPES in undoped materials, i.e., the single hole Lehman spectral function (LSF) doped into the Mott insulator, [8] has recently been understood in terms of the small polaron formation 9, 10, 11]. It has been revealed that the energy dispersion of the extended $t-J$ model, $t t^{\prime} t^{\prime \prime}-J$ model 12 survives as the center of mass position of the broad Franck-Condon peak (FCP) of phonon side-bands even at strong EPI, while the weight $Z$ of the zero-phonon line and its dispersion is very small. Recently, decoupling of chemical potential from observed in ARPES resonance, predicted by strong EPI scenario, was confirmed experimentally [5] and, thus, phonon origin of ARPES broadening seems to be likely.

In addition to high- $T_{c}$ problem, strong EPI mechanism of ARPES spectra broadening was considered as one of alternative scenarios for diatomic molecules 13], colossal magnetoresistive manganites 14], quasi-one-dimensional Peierls conductors [15], and Verwey magnetites [16]. Therefore, exact analysis of the IE on ARPES at strong EPI is of general interest for conclusive experiments in a broad variety of compound classes.

In this Letter we present a study of the IE on the single particle LSF of a hole strongly interacting with phonons in the $t t^{\prime} t^{\prime \prime}-J$ model, which is equivalent to study of ARPES in undoped cuprates [4]. As mentioned above, it is essential to compare experiment in undoped systems with the present DMC results, where theory can offer quantitative approximation-free results. We calculate IE on LSF by exact DMC method at zero temperature in nodal $(\pi / 2, \pi / 2)$ and antinodal $(\pi, 0)$ points of the Brillouin zone for realistic parameters of the $t t^{\prime} t^{\prime \prime}-J$ model in the intermediate and strong EPI regime. We find that IE is anomalously enhanced in the intermediate EPI regime but approaches simple analytic estimates in the strong EPI regime. We analyze the features of the FCP which are most sensitive to IE at different EPI regimes.

In the standard spin-wave approximation for twodimensional $t t^{\prime} t^{\prime \prime}-J$ model, which was shown to be a good approximation for small exchange integrals $J / t \leq 0.4$ 17], a hole with dispersion [12] $\varepsilon(\mathbf{k})=$ $4 t^{\prime} \cos \left(k_{x}\right) \cos \left(k_{y}\right)+2 t^{\prime \prime}\left(\cos \left(2 k_{x}\right)+\cos \left(2 k_{y}\right)\right)$ propagates in the magnon and phonon (annihilation operators are $\alpha_{\mathbf{k}}$ and $b_{\mathbf{k}}$, respectively) bathes

$$
\hat{H}_{\mathrm{t}-\mathrm{J}}^{0}=\sum_{\mathbf{k}} \varepsilon(\mathbf{k}) h_{\mathbf{k}}^{\dagger} h_{\mathbf{k}}+\sum_{\mathbf{k}} \omega_{\mathbf{k}} \alpha_{\mathbf{k}}^{\dagger} \alpha_{\mathbf{k}}+\Omega \sum_{\mathbf{k}} b_{\mathbf{k}}^{\dagger} b_{\mathbf{k}}
$$

with magnon dispersion $\omega_{\mathbf{k}}=2 J \sqrt{1-\gamma_{\mathbf{k}}^{2}}$, where $\gamma_{\mathbf{k}}=$ 
$\left(\cos k_{x}+\cos k_{y}\right) / 2$. The hole is scattered by magnons

$$
\hat{H}_{\mathrm{t}-\mathrm{J}}^{\mathrm{h}-\mathrm{m}}=N^{-1 / 2} \sum_{\mathbf{k}, \mathbf{q}} M_{\mathbf{k}, \mathbf{q}}\left[h_{\mathbf{k}}^{\dagger} h_{\mathbf{k}-\mathbf{q}} \alpha_{\mathbf{q}}+h . c .\right],
$$

where $M_{\mathbf{k}, \mathbf{q}}$ is the standard vertex [17]. We chose the simplest Holstein short-range EPI Hamiltonian

$$
\hat{H}^{\mathrm{e}-\mathrm{ph}}=N^{-1 / 2} \sum_{\mathbf{k}, \mathbf{q}} \frac{\sigma}{\sqrt{2 M \Omega}}\left[h_{\mathbf{k}}^{\dagger} h_{\mathbf{k}-\mathbf{q}} b_{\mathbf{q}}+h . c .\right],
$$

where $\sigma$ is the momentum and isotope independent coupling constant, $M$ is the mass of the vibrating lattice ions, and $\Omega$ is the frequency of dispersionless phonon (Planck constant $\hbar$ is set to unity). We introduce dimensionless coupling constant $\lambda=\gamma^{2} / 4 t \Omega$ which is, in contrast to the

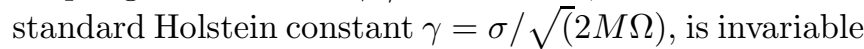
quantity for the simplest case of IE. Indeed, assuming the simplest natural relation $\Omega \sim 1 / \sqrt{M}$ between phonon frequency and mass, we find that $\lambda$ does not depend on the isotope factor $\kappa_{\text {iso }}=\Omega / \Omega_{0}=\sqrt{M_{0} / M}$, which is defined as the ratio of phonon frequency in isotope substituted $\Omega$ and normal $\Omega_{0}$ system.

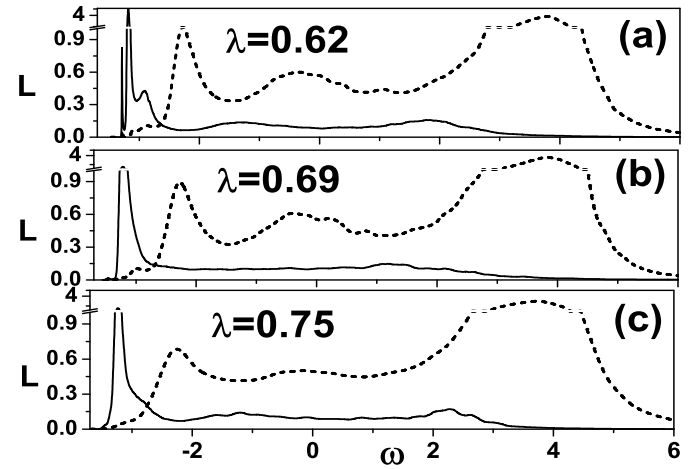

FIG. 1: Hole LSFs at $\mathbf{k}=(\pi / 2, \pi / 2)$ (solid line) and $\mathbf{k}=$ $(\pi, 0)$ (dashed line) for different couplings.

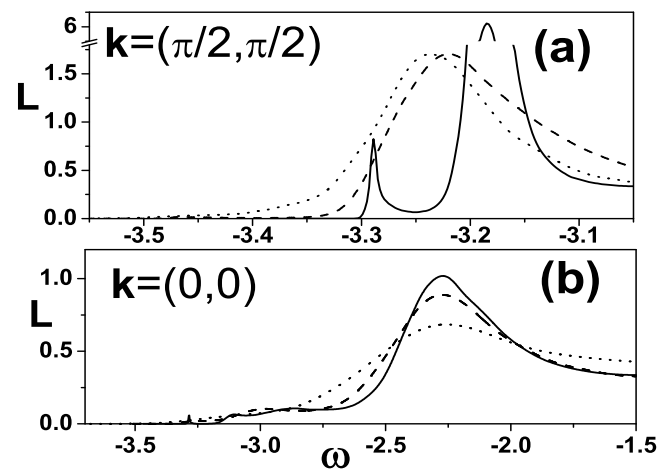

FIG. 2: Low energy part of LSF for $\lambda=0.62$ (solid line), $\lambda=0.69$ (dashed line), and $\lambda=0.75$ (dotted line) at nodal (a) and antinodal (b) points.

We chose adopted parameters of the $t t^{\prime} t^{\prime \prime}-J$ model which reproduce the experimental dispersion of a quasiparticle (QP) [12]: $J / t=0.4, t^{\prime} / t=-0.34$, and $t^{\prime \prime} / t=$
0.23 . The frequency of the relevant phonon [4] is set to $\Omega / t=0.2$ and the isotope factor $\kappa_{\text {iso }}=\sqrt{16 / 18}$ corresponds to substitution of $\mathrm{O}^{18}$ isotope for $\mathrm{O}^{16}$.

We use DMC method [18, 19], which is the only available method for approximation-free study of excited states of problem (13) for the macroscopic system at strong EPI. Non-crossing approximation for phonon variables 20] is shown to be invalid approximation for strong EPI 9] while exact diagonalization studies of small clusters, although account for correct treatment of phonons, imply a discrete spectrum and, thus, tiny changes of the FCP lineshape for $\kappa \approx 1$ are not reliable. The only study of IE by exact method 21], i.e. path integral quantum Monte Carlo algorithm 22], does not addresses realistic $t t^{\prime} t^{\prime \prime}-J$ model, where both phonon and magnon variables have to be summed simultaneously and does not study ARPES directly. On the other hand, DMC method allows to treat ARPES directly [9]. We use recently developed Stochastic Optimization method [18] which avoids regularization and artificial broadening of LSF peaks. To sweep aside any doubts of possible instabilities of analytic continuation, we calculate the LSF for normal compound $\left(\kappa_{\text {nor }}=1\right)$, isotope substituted $\left(\kappa_{\text {iso }}=\sqrt{16 / 18}\right)$ and "anti-isotope" substituted $\left(\kappa_{\text {ant }}=\sqrt{18 / 16}\right)$ compounds. Monotonic dependence of LSF on $\kappa$ ensures stability of analytic continuation and gives possibility to evaluate the error-bars of a quantity $\mathcal{A}$ using quantities $\mathcal{A}_{\text {iso }}-\mathcal{A}_{\text {nor }}$, $\mathcal{A}_{\text {nor }}-\mathcal{A}_{\text {ant }}$, and $\left(\mathcal{A}_{\text {iso }}-\mathcal{A}_{\text {ant }}\right) / 2$.

Figure 1 presents LSFs in nodal and antinodal points for values of EPI which are larger than the critical self-trapping (ST) coupling $\lambda_{\text {cr }} \approx 0.58$, i.e. coupling where fast transformation to regime of strong EPI occurs 23, 24]. Since LSF is sensitive to strengths of EPI only for low frequencies we concentrate on the low energy part of the spectrum (Fig. (2). It is seen that near the ST crossover the LSF in the nodal point quickly changes with coupling while FCP in antinodal point gradually broadens. The bandwidth of FCP dispersion is estimated as $W_{\mathrm{FCP}} \approx 0.9$.

Figures 3 and 4 show IE on the hole LSF for different couplings in nodal and antinodal points, respectively. The general trend is a shift of all spectral features to larger energies with increase of the isotope mass $(\kappa<1)$. One can also note that the shift of broad FCP is much larger than that of real narrow QP peak. Moreover, for large couplings $\lambda$ the shift of QP energy approaches zero and only decrease of QP spectral weight $Z$ is observed for larger isotope mass. On the other hand, the shift of FCP is not suppressed for larger couplings. Except for the LSF in nodal point at $\lambda=0.62$ (Fig. 3a, b), where LSF still has a notable weight of QP $\delta$-functional peak, there is one more notable feature of the IE. With increase of the isotope mass the height of FCP increases. Taking into account the conservation law for LSF $\int_{-\infty}^{+\infty} L_{\mathbf{k}}(\omega)=1$ and insensitivity of high energy part of LSF to EPI strength (Fig. 2), the narrowing of the FCP for larger isotope mass can be concluded. To understand the trends of the IE in the strong coupling 
regime we analyze the exactly solvable independent oscillators model (IOM) 25]. More rigorous Lang-Firsov transformation is not required since it is not valid for adiabatic case $\Omega / t=0.2$ and in the intermediate coupling regime 21] while in the strong coupling regime, as it is shown below, IOM shows quantitative agreement with DMC data. Indeed, coherent QP bandwidths $W_{\mathrm{QP}}(\lambda=0.62) \approx 3.2 * 10^{-3}, W_{\mathrm{QP}}(\lambda=0.69) \approx 6 * 10^{-4}$, and $W_{\mathrm{QP}}(\lambda=0.75) \approx 4 * 10^{-4}$ are negligibly small in comparison with FCP bandwidth $W_{\mathrm{FCP}} \approx 0.9$. The

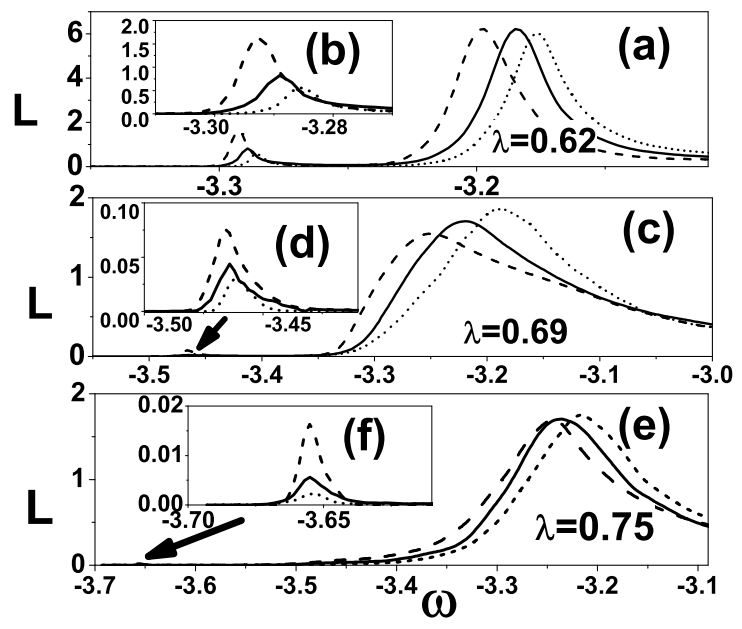

FIG. 3: Low energy part of hole LSFs in the nodal point at different couplings (a, c, e): normal compound (solid line), isotope substituted compound (dotted line) and "antiisotope" substituted compound (dashed line). Insets (b, d, e) show low energy real QP peak.
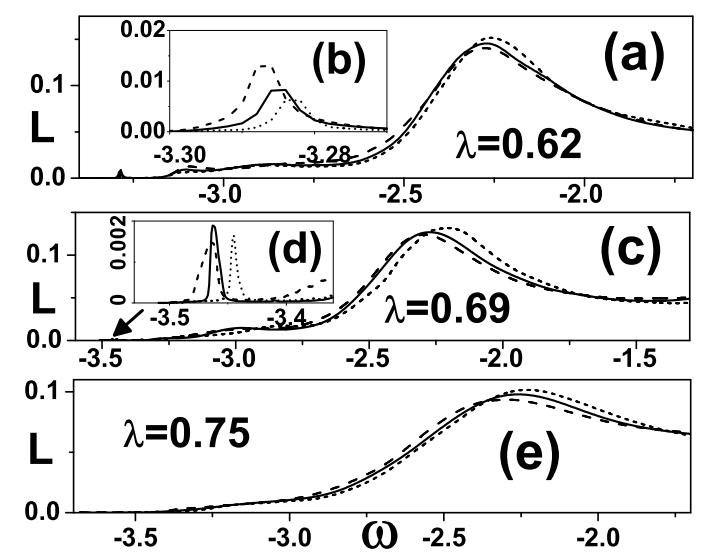

FIG. 4: Low energy part of hole LSFs in the antinodal point. See caption of Fig. [3]

LSF in IOM is the Poisson distribution

$$
L(\omega)=\exp \left[-\xi_{0} / \kappa\right] \sum_{l=0}^{\infty} \frac{\left[\xi_{0} / \kappa\right]^{l}}{l !} \mathcal{G}_{\kappa, l}(\omega)
$$

where $\xi_{0}=\gamma_{0}^{2} / \Omega_{0}^{2}=4 t \lambda / \Omega_{0}$ is dimensionless coupling constant for normal system and $\mathcal{G}_{\kappa, l}(\omega)=\delta[\omega+4 t \lambda-$

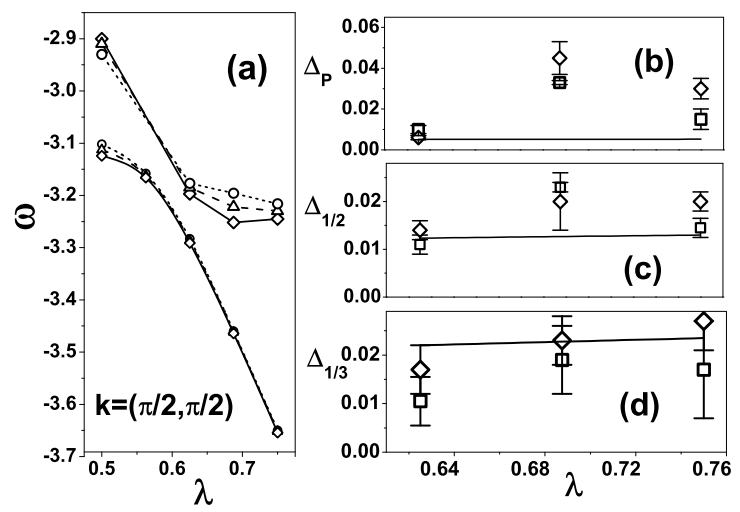

FIG. 5: Energies of ground state and broad peaks (a) for normal (tringles), isotope substituted (circles) and "antiisotope" substituted (dimonds) compounds. Comparison of IOM estimates (lines) with DMC data in the nodal (squares) and antinodal (diamonds) points: shift of the FCP top (b), FCP leading edge at $1 / 2$ of height (c), and FCP leading edge at $1 / 3$ of height $(d)$.

$\left.\Omega_{0} \kappa l\right]$ is the $\delta$-function. The properties of the Poisson distribution quantitatively explain many features of the IE on LSF.

The energy $\omega_{\mathrm{QP}}=-4 t \lambda$ of the zero-phonon line $l=0$ in (4) depends only on isotope independent quantities which explains very weak isotope dependence of QP peak energy in insets of Figs. 34 Besides, change of the zero-phonon line weight $Z^{(0)}$ obeys relation $Z_{\text {iso }}^{(0)} / Z_{\text {nor }}^{(0)}=$ $\exp \left[-\xi_{0}(1-\kappa) / \kappa\right]$ in IOM. These IOM estimates agree with DMC data within $15 \%$ in the nodal point and within $25 \%$ in the antinodal one. IE on FCP in the strong coupling regime follows from the properties of zero $M_{0}=\int_{-\infty}^{+\infty} L(\omega) d \omega=1$, first $M_{1}=\int_{-\infty}^{+\infty} \omega L(\omega) d \omega=0$, and second $M_{2}=\int_{-\infty}^{+\infty} \omega^{2} L(\omega) d \omega=\kappa \xi_{0} \Omega_{0}^{2}$ moments of shifted Poisson distribution (4). Moments $M_{0}$ and $M_{2}$ establish relation $\mathcal{D}=h_{\text {iso }}^{\mathrm{FCP}} / h_{\text {nor }}^{\mathrm{FCP}}=1 / \sqrt{\kappa} \approx 1.03$ between heights of FCP in normal and substituted compounds. DMC data in the antinodal point perfectly agree with the above estimate for all couplings. This is consistent with the idea that the anti-nodal region remains in the strong coupling regime even though the nodal region is in the crossover region. In the nodal point $\mathrm{DMC}$ data well agree with IOM estimate for $\lambda=0.75(\mathcal{D} \approx 1.025)$ whereas at $\lambda=0.69$ and $\lambda=0.62$ influence of the ST point leads to anomalous values of $\mathcal{D}: \mathcal{D} \approx 1.07$ and $\mathcal{D} \approx 0.98$, respectively. Shift of the low energy edge at half maximum $\Delta_{1 / 2}$ must be proportional to change of the root square of second moment $\Delta_{\sqrt{M_{2}}}=\sqrt{\xi_{0}} \Omega_{0}[1-\sqrt{\kappa}]$. As we found in numeric simulations of Eq. (4) with Gaussian functions [27] $\mathcal{G}_{\kappa, l}(\omega)$, relation $\Delta_{1 / 2} \approx \Delta_{\sqrt{M_{2}}} / 2$ is accurate to $10 \%$ for $0.62<\lambda<0.75$. Also, simulations show that the shift of the edge at one third of maximum $\Delta_{1 / 3}$ obeys relation $\Delta_{1 / 3} \approx \Delta_{\sqrt{M_{2}}}$. DMC data with IOM estimates are in good agreement for strong EPI $\lambda=0.75$ (Fig. 515). However, shift of the FCP top $\Delta_{p}$ and $\Delta_{1 / 2}$ are 
considerably enhanced in the self-trapping (ST) transition region. The physical reason for enhancement of IE in this region is general property regardless of the QP dispersion, range of EPI, etc. The influence of nonadiabatic matrix element, mixing excited and ground states, on the energies of resonances essentially depends on the phonon frequency. While in the adiabatic approximation ST transition is sudden and nonanalytic in $\lambda$ [23], nonadiabatic matrix elements turn it to smooth crossover 26]. Thus, as illustrated in Fig. 5 , the smaller the frequency the sharper the kink in the dependence of excited state energy on the interaction constant.

Cautions should be made about approximate form of EPI (3). Strictly speaking, actual momentum dependence of the interaction constant $\sigma$ [28, 29] can slightly change the obtained differences between nodal and antinodal points though the general trends have to be left intact because ST is caused solely by the short range part of EPI 23]. Also the shell model calculation [28] has shown that the dominant EPI is the short range one associated with the oxygen displacements. Therefore, even though the participation of $\mathrm{Cu}$ ions [28] in the lattice vibrations relevant to EPI violates simple relation $\Omega \sim 1 / \sqrt{M}$, this relation is approximately valid.

Finally, we discuss the relevance of the present results to the isotope experiment on the doped high $T_{c}$ cuprates 7]. The most puzzling feature is that the IE is negligible near the phonon frequency, and is mainly observed in the high energy region. This can not be explained by the conventional Eliashberg-Migdal theory, which predicts the suppression of the multiphonon emission at high energy and the IE only near the one-phonon frequency. The intermediate and strong coupling polaron theory gives a completely different picture, where the high energy part consists of the multiphonon sidebands and is subject to the IE as shown in this paper. Although it is not clear at the moment when the crossover from the doped hole picture to the metallic large Fermi surface occurs as the doping proceeds, the IE experiment [7] suggests the former might persists even at the optimal doping. However more study is needed both theoretically and experimentally for the doped case. In the undoped case, on the other hand, the present results can be directly compared with the experiments and more solid studies can be done. It is found that isotope effect on the ARPES lineshape of a single hole is anomalously enhanced in the intermediate coupling regime while can be described by simple independent oscillators model in the strong coupling regime. The shift of FCP top and change of the FCP height are relevant quantities to pursue experimentally in the intermediate coupling regime since IE on these characteristics is enhanced near the self trapping point. In contrast, shift of the leading edge is the relevant quantity in the strong coupling regime since this value increases with coupling as $\sqrt{\lambda}$. These conclusions, depending on the fact whether self trapping phenomenon is encountered in specific case, can be applied fully or partially to another compounds with strong EPI [14, 15, 16].

Note added in proof. Related studies of the isotope effect on ARPES were recently reported by S. Fratini and S. Ciuchi [30] in the framework of dynamical mean field approach to the Mott-Hubbard insulator and by G. Seibold and M. Grilli 31] where coupling of quasiparticle to a charge collective mode is treated within the simple perturbative scheme.

Fruitful discussions with G.-H. Gweon are acknowledged. This work was supported by RFBR 04-0217363a, and the Grants-in-aid for Scientific Research and NAREGI Nanoscience Project from the Ministry of Education, Culture, Sports, Science, and Technology.
[1] P. W. Andreson, The Theory of Superconductivity in the High- $T_{c}$ Cuprates (Princeton University Press, New Jersey, 1997).

[2] D. S. Marshall, D. S. Dessau, A. G. Loeser, C-H. Park, A. Y. Matsuura, J. N. Eckstein, I. Bozovic, P. Fournier, A. Kapitulnik, W. E. Spicer, and Z.-X. Shen, Phys. Rev. Lett. 76, 4841 (1996).

[3] M. R. Norman, H. Ding, M. Randeria, J.C. Campuzano, T. Yokoya, T. Takeuchi, T. Takahashi, T. Mochiku, K. Kadowaki, P. Guptasarma, and D.G Hinks, Nature 392, 157 (1998)

[4] A. Danmascelli, Z.-X. Shen, and Z. Hussain, Rev. Mod. Phys. 75, 473 (2003).

[5] K. M. Shen, F. Ronning, D. H. Lu, W. S. Lee, N. J. C. Ingle, W. Meevasana, F. Baumberger, A. Damascelli, N. P. Armitage, L. L. Miller, Y. Kohsaka, M. Azuma, M. Takano, H. Takagi, and Z.-X. Shen, Phys. Rev. Lett. 93, 267002 (2004).

[6] K. M. Shen, F. Ronning, D. H. Lu, F. Baumberger, N. J. C. Ingle, W. S. Lee, W. Meevasana, Y. Kohsaka, M. Azuma, M. Takano, H. Takagi, and Z.-X. Shen, Science
307, 901 (2005).

[7] G.-H. Gweon, T. Sasagawa, S. Y. Zhou, J. Graf, H. Takagi, D. H. Lee, and A. Lanzara, Nature 430, 187 (2004).

[8] B. O. Wells, Z.-X. Shen, A. Matsuura, D. M. King, M. A. Kastner, M. Greven, and R. J. Birgeneau, Phys. Rev. Lett. 74, 964 (1995).

[9] A. S. Mishchenko and N. Nagaosa, Phys. Rev. Lett.93, 036402 (2004).

[10] O. Rösch, O. Gunnarsson, X. J. Zhou, T. Yoshida, T. Sasagawa, A. Fujimori, Z. Hussain, Z.-X. Shen, and S. Uchida, Phys. Rev. Lett. 95, 227002 (2005).

[11] O. Rösch and O. Gunnarsson, Eur. Phys. J B 43, 11 (2005).

[12] T. Xiang and M. Wheatley, Phys. Rev. B 54, R12653 (1996).

[13] G. A. Sawatzky, Nature (London) 342B, 480 (1989).

[14] D. S. Dessau, T. Saitoh1, C.-H. Park, Z.-X. Shen, P. Villella, N. Hamada, Y. Moritomo, and Y. Tokura, Phys. Rev. Lett. 81, 192 (1998); V. Perebeinos and P. B. Allen, ibid. 85, 5178 (2000). N. Mannella, A. Rosenhahn, C. H. Booth, S. Marchesini, B. S. Mun, S.-H. Yang, K. Ibrahim, 
Y. Tomioka, and C. S. Fadley, ibid. 92, 166401 (2004).

[15] L. Perfetti,H. Berger, A. Reginelli, L. Degiorgi, H. Hchst, J. Voit, G. Margaritondo, and M. Grioni, Phys. Rev. Lett. 87, 216404 (2001); L. Perfetti, S. Mitrovic, G. Margaritondo, M. Grioni, L. Forró, L. Degiorgi, and H. Höchst, Phys. Rev. B 66, 075107 (2002).

[16] D. Schrupp, M. Sing, M. Tsunekawa, H. Fujiwara, S. Kasai, A. Sekiyama, S. Suga, T. Muro, V. A. M. Brabers and R. Claessen, Eur. Phys. Lett. 70, 789 (2005).

[17] C. L. Kane, P. A. Lee, and N. Read, Phys. Rev. B 39, 6880 (1989); Z. Liu and E. Manousakis, ibid. 45, 2425 (1992).

[18] A. S. Mishchenko, N. V. Prokof'ev, A. Sakamoto, and B. V. Svistunov, Phys. Rev. B 62, 6317 (2000).

[19] A. S. Mishchenko and N. Nagaosa, Phys. Rev. Lett. 86, 4624 (2001); A. S. Mishchenko, N. Nagaosa, N. V. Prokof'ev, A. Sakamoto, and B. V. Svistunov, ibid. 91, 236401 (2003); A. S. Mishchenko, Physics-Uspekhi 48, 887 (2005).

[20] A. Ramsak, P. Horsch, and P. Fulde, Phys. Rev. B 46, 14305 (1992); B. Kyung, S. I. Mukhin, V. N. Kostur, and R. A. Ferrell, ibid. 54, 13167 (1996).

[21] P. E. Kornilovitch and A. S. Alexandrov, Phys. Rev. B
70, 224511 (2004).

[22] P. E. Spenser, J. H. Samson, P. E. Kornilovitch, and A. S. Alexandrov, Phys. Rev. B 71, 184310 (2005).

[23] E. I. Rashba, in Modern Problems in Condensed Matter Sciences, Ed. by V. M. Agranovich and A. A. Maradudin (North-Holland, Amsterdam, 1982), Vol. 2, p. 543.

[24] A.S. Mishchenko, N. Nagaosa, N. V. Prokof'ev, A. Sakamoto, and B. V. Svistunov, Phys. Rev. B 66, 020301 (2002).

[25] G. D. Mahan, Many Particle Physics (Plenum Press, New york, 1990).

[26] B. Gerlach and H. Löwen, Rev. Mod. Phys. 63, 63 (1991).

[27] Results are almost independent on the parameter $\eta$ of the Gausssian distribution $\mathcal{G}_{\kappa, l}(\omega)=1 /(\eta \sqrt{2 \pi}) \exp (-[\omega+$ $\left.\left.4 t \lambda-\Omega_{0} \kappa l\right] /\left(2 \eta^{2}\right)\right)$ in the range $[0.12,0.2]$.

[28] O. Rösch and O. Gunnarsson, Phys. Rev. Lett. 92, 146403 (2004).

[29] S. Ishihara and N. Nagaosa, Phys. Rev. B 69, 144520 (2004).

[30] S. Fratini and S. Ciuchi, Phys. Rev. B 72, 235107 (2005).

[31] G. Seibold and M. Grilli, Phys. Rev. B 72, 104519 (2005). 